\title{
Interactions of a hemidesmosome component and actinin family members
}

\author{
Annette M. Gonzalez¹, Carol Otey ${ }^{2}$, Magnus Edlund ${ }^{2}$ and Jonathan C. R. Jones, ${ }^{1, *}$ \\ ${ }_{1}^{1}$ Department of Cell and Molecular Biology, Northwestern University Medical School, 303 E. Chicago Avenue, Chicago, IL 60611, USA \\ 2Department of Cell and Molecular Physiology, University of North Carolina, Chapel Hill, NC 27599, USA \\ *Corresponding author (e-mail: j-jones3@nwu.edu) \\ Accepted 31 August 2001 \\ Journal of Cell Science 114, 4197-4206 (2001) (C) The Company of Biologists Ltd
}

\section{SUMMARY}

Hemidesmosomes are multimeric protein complexes that attach epithelial cells to their underlying matrix and serve as cell surface anchorage sites for the keratin cytoskeleton. Two hemidesmosome components, the $\alpha 6 \beta 4$ integrin heterodimer and a human autoantigen termed BP180, are transmembrane proteins that link the extracellular matrix to the keratin network in cells. Here, we report that actinin4 , an actin-bundling protein, is a potential binding partner for BP180. Using yeast two-hybrid, we have mapped the binding site for BP180 to the C-terminal region of actinin4. This site contains two $\mathrm{EF}$-hand, $\mathrm{Ca}^{2+}$ regulation domains and shares $87 \%$ sequence homology with the same region in actinin-1. Consistent with this, BP180 can bind actinin-1 in both the yeast two-hybrid assay and in immunoprecipitation assays. To determine whether the EFhand domain is a consensus binding sequence for BP180, we tested whether other proteins with this domain bind BP180. None of the proteins tested including calmodulin, with 4 EF-hand domains, and myosin regulatory light chain, with $1 \mathrm{EF}$-hand domain, interacts with BP180 in yeast two-hybrid system and immunoprecipitation studies, suggesting that the interaction between BP180 and actinin family members is specific. We have compared the distribution of actinin-1 and actinin-4 with that of BP180 in MCF-10A and pp126 cells. Surprisingly, BP180 localizes not only to sites of cell-substratum interaction, but is also present at sites of cell-cell contacts where it co-distributes with both actinin-1 and actinin- 4 as well as other adherens junction proteins. In oral tissues, BP180 is present along the basement membrane and at cell-cell contact sites in basal epithelial cells where it co-distributes with adherens junction proteins. Since BP180 antibodies inhibit association of junction proteins at sites of cell-cell contact in oral keratinocytes, these results suggest that BP180 may play a role in establishing cell-cell interactions. We discuss a role for BP180 in crosstalk between cell-matrix and cellcell junctions.

Key words: Human autoantigen, hemidesmosomes, actin cytoskeleton

\section{INTRODUCTION}

The hemidesmosome is a multimeric protein complex that provides stable cell attachment to the underlying matrix of epithelial cells (Borradori and Sonnenberg, 1999; Jones et al., 1998). At the ultrastructure level, the hemidesmosome appears as an electron dense structure whose cytoplasmic plaque serves to anchor keratin-type intermediate filaments to the cell surface (Borradori and Sonnenberg, 1999; Jones et al., 1998). A number of components of the hemidesmosome have been identified and these include at least two cytoskeleton linker proteins, a $230 \mathrm{kDa}$ human autoantigen termed BP230 (BPAG1) and plectin, as well as two transmembrane protein complexes, the $\alpha 6 \beta 4$ integrin heterodimer and a second human autoantigen called BP180 (BPAG2) (Borradori and Sonnenberg, 1999; Jones et al., 1998; Klatte et al., 1989; Rezniczek et al., 1998).

BP180, also known as collagen XVII, was first identified using sera of patients suffering from bullous pemphigoid, a blistering skin disease (Giudice et al., 1992; Jones et al., 1998). It is a type 2 transmembrane protein whose $\mathrm{N}$-terminus resides in the cytoplasmic plaque of the hemidesmosome (Hopkinson et al., 1992). The extracellular domain of BP180 is composed of a series of collagen-like repeats that are separated by linker regions (Giudice et al., 1992; Li et al., 1993). Like other collagens, BP180 exists as a trimer and it has now been established that the extracellular domain of BP180 contributes to the structure of anchoring filaments observed in the lamina lucida of the basement membrane zone (Balding et al., 1997; Bedane et al., 1997; Hirako et al., 1996).

Evidence from analyses of the skin of patients suffering from bullous pemphigoid and generalized atrophic epidermolysis bullosa (GABEB), a genetic blistering skin disease, indicate that BP180 plays an important role in hemidesmosome assembly and adhesion of the epidermis to the dermis (Borradori and Sonnenberg, 1999; Jonkman et al., 1995). In bullous pemphigoid, autoantibodies against a noncollagenous extracellular epitope in the BP180 molecule are believed to be pathogenic and induce dysadhesion of the epidermis (Giudice et al., 1993; Liu et al., 1993). Moreover, epidermal cells in GABEB patients carrying mutations in the gene that encodes for BP180 have a reduction in the number of hemidesmosomes, leading to tissue instability (Borradori and Sonnenberg, 1999; Jonkman et al., 1995).

BP180 has multiple binding partners in the hemidesmosome. The cytoplasmic domain of BP180 can interact with BP230 
and the $\beta 4$ integrin subunit, whereas the extracellular domain can interact with the $\alpha 6$ integrin subunit (Aho and Uitto, 1998; Borradori et al., 1997; Hopkinson et al., 1998; Hopkinson and Jones, 2000). In this study, we focused our attention on identifying novel binding partners of the cytoplasmic domain of the BP180 molecule. Using yeast two-hybrid technology we have identified actinins as binding partners of BP180. This finding was surprising since BP180 had been thought to be present exclusively at hemidesmosomes, where it links to intermediate filaments. Conversely, the actinins are microfilament-associated proteins that are found in adherens junctions at sites of cell-cell interaction and in focal contacts at sites where cells abut their substrate (Borradori and Sonnenberg, 1999; Burridge and Chrzanowska-Wodnicka, 1996; Jones et al., 1998). However, in this study we show that BP180 is not only found at sites of cell-substrate interaction but also is concentrated at regions of cell-cell-contact, where it co-distributes with and can interact with a number of adherens junction proteins. Moreover, we present evidence that BP180 is involved in establishment of intercellular junctions and can play a role in cell-cell interactions.

\section{MATERIALS AND METHODS}

\section{Cell culture and transfection procedure}

MCF-10A and pp126 cells were cultured as detailed previously (Goldfinger et al., 1998). Cells were maintained for 72 hours on 22 $\mathrm{mm}$ glass coverslips. They were transfected with $4 \mu \mathrm{g}$ of plasmid DNA using the calcium phosphate protocol (Sambrook et al., 1989). At 24 hours following transfection cells were harvested for immunoblotting or processed for immunofluorescence microscopy (see below).

\section{Yeast two-hybrid assay}

An MCF-10A cDNA library was prepared according to the TwoHybrid cDNA Library Construction Kit protocol (Clontech Labs Inc., Palo Alto, CA). cDNAs encoding portions of BP180, actinin family members, calmodulin and $\alpha 6$-integrin were amplified using RT-PCR from MCF10A mRNA using specific forward and reverse primers containing engineered restriction sites. Murine myosin regulatory light chain (MRLC) was a gift from Rex Chisholm (Northwestern University). These fragments were digested with the appropriate enzymes, isolated from an agarose gel using the QIAquick gel extraction kit (Qiagen, Charworth, CA), and ligated in frame into digested yeast expression vectors pACT2 or pAS2-1 (Clontech Labs Inc., Palo Alto, CA). Individual clones containing these constructs were grown in selective media and DNA prepared from the clones using a Wizard mini prep kit (Promega, Madison, WI). All constructs were sequenced to ensure that the cDNAs were in frame and without error using Big Dye automated sequencing reagents (PE Applied Biosystems, Foster City, CA) on an ABI Prism DNA Sequencer (Foster City, CA).

DNA preparations were used to transform the yeast strain Y190, according to protocols outlined in the Matchmaker Two-Hybrid System 2 Manual (Clontech Labs). Transfected colonies were selected by growth in medium lacking leucine, tryptophan and histidine ( $\left.\mathrm{Leu}^{-} / \mathrm{Trp}^{-} / \mathrm{His}^{-}\right)$but containing $25 \mathrm{mM}$ 3-amino-1,2,4-triazole (3AT). The latter was used to inhibit low levels of 'leaky' expression of His $3 p$ in the reporter yeast strain. To monitor transfection efficiency of both plasmids, the transfected yeast were also plated onto $\mathrm{Leu}^{-}$ medium, Trp ${ }^{-}$medium or $\mathrm{Leu}^{-} / \mathrm{Trp}^{-}$medium. At 7 days the number of colonies growing on both the $\mathrm{Leu}^{-} / \mathrm{Trp}^{-}$and $\mathrm{Leu}^{-} / \mathrm{Trp}^{-} / \mathrm{His}^{-}$media were scored. Yeast colonies growing on both $\mathrm{Leu}^{-} / \mathrm{Trp}^{-} / \mathrm{His}^{-}$and
$\mathrm{Leu}^{-} / \mathrm{Trp}^{-}$media were also spotted onto nylon filters and flash frozen in liquid nitrogen. To detect activation of the reporter gene lacZ and resulting expression of $\beta$-galactosidase, the filters were placed on Whatman paper soaked in a solution containing X-gal (5-bromo-4chloro-3-indolyl- $\beta$-D-galactopyranoside). A binding domain plasmid (pVA3-1), containing a cDNA encoding murine $\mathrm{p} 53$, and an activation domain plasmid (pTD1-1), containing the SV40 large T-antigen coding sequence, were used as part of our control studies (Clontech Labs).

\section{Recombinant protein preparation}

The cytoplasmic domain of BP180 (residues 1-461), actinin-4 (residues 1-884), actinin-1 (residues 1-892) and MRLC (residues 1172) were prepared as $6 \times$ His-tagged recombinant fusion proteins in E. coli. The relevant portion of BP180 was generated by RT-PCR using MCF10A mRNA and the BP180 cDNA fragment was cloned in frame into the pET 32 vector (Novagen, Madison, WI). This construct was sequenced to confirm that the reading frame was maintained and that the sequence was correct. E. coli were transformed with the BP180 construct and were subsequently induced to produce recombinant protein by the addition of isopropyl- $\beta-D-$ thiogalactopyranoside (IPTG) to the medium. The cells were lysed and extracts were incubated overnight in a $6 \mathrm{M}$ urea buffer. Cell extracts were passed over a His.Bind Resin column (Novagen, Madison, WI) and bound fusion protein eluted in an imidazole elution buffer in the presence of $6 \mathrm{M}$ urea. The eluant was dialyzed against $10 \mathrm{mM}$ Tris buffer $(\mathrm{pH} 7.5)$ overnight at $4^{\circ} \mathrm{C}$, concentrated by lyophilization and resuspended in sterile $\mathrm{H}_{2} \mathrm{O}$. The purity of the recombinant polypeptides was assessed by visualizing the protein samples by SDS-PAGE and by Western blotting.

To generate HA-tagged proteins, actinin-4, actinin-1 and MRLC, pACT2 constructs were used as DNA templates since the pACT2 vector contains a hemagglutinin sequence (HA) upstream of the cloning site. Vector-specific forward and sequence-specific reverse primers were used to generate cDNAs for actinin-4, actinin- 1 and MRLC containing the HA sequence at the $5^{\prime}$ end. The PCR products were purified and cloned in frame into the pBAD-TOPO vector (Invitrogen, Carlsbad, CA). Constructs were sequenced as described above to ensure that sequences were correct and that inserts were in frame. E. coli were transformed with appropriate constructs and expression of recombinant protein was induced by adding $2 \%$ arabinose to the medium for 3 hours at $37^{\circ} \mathrm{C}$. Cells were lysed, centrifuged and the supernatant was passed over a His Bind Resin column (Novagen). Proteins were eluted in an imidazole elution solution and eluates were dialyzed overnight and processed as described above.

\section{Green fluorescent protein (GFP) and HA-tagged constructs}

cDNA's encoding full-length actinin-4 (residues 1-884) and truncated actinin-4 (residues 1-813) with the HA tag incorporated at $5^{\prime}$ end, were cloned into pCR3.1-Uni vector (Invitrogen). In addition, actinin4 cDNA cloned into the pEGFP-NI vector (Clontech) was used in some transfection studies.

\section{Antibodies}

A mouse IgM monoclonal antibody (1804b) against the N-terminal domain of BP180 was prepared as described (Hopkinson et al., 1992; Riddelle et al., 1992). J17 rabbit antiserum was generated against the same BP180 domain (Hopkinson et al., 1992). The rabbit C-terminal antiserum generated against the C-terminal domain of BP180 was obtained from Kim Yancey (NIH, Bethesda, MD). Monoclonal antibody HA.11 against the HA epitope tag was obtained from BAbCO. The actinin-4 polyclonal serum was a generous gift from Setsuo Hirohashi (Honda et al., 1998). The mouse monoclonal antibody against actinin-1 was purchased from Sigma Chemical Co. The p120 antibody was obtained from BD Transduction Laboratories. 
The E-cadherin monoclonal antibody was generously provided by Kathy Green (Northwestern University).

\section{Gel electrophoresis, immunoblotting and immunoprecipitation}

Recombinant proteins, bacterial and mammalian cell extracts were solubilized in sample buffer ( $8 \mathrm{M}$ urea, $10 \% \beta$-mercaptoethanol, $1 \%$ SDS, $10 \%$ glycerol in $10 \mathrm{mM}$ Tris- $\mathrm{HCl}, \mathrm{pH}$ 6.8) and were subjected to SDS-PAGE using 7.5-10\% acrylamide gels (Laemmli, 1970). For western immunoblotting, proteins separated on gels were transferred to nitrocellulose membranes that were then processed with antibody as described elsewhere (Harlow and Lane, 1988). S-tag peptide fusion proteins were visualized with $\mathrm{S}$ protein conjugated to alkaline phosphatase (Novagen).

For immunoprecipitation studies using recombinant proteins, approximately $1 \mu \mathrm{g}$ of actinin-4, actinin- 1 or MRLC protein and BP180 fragments were mixed together in $500 \mu \mathrm{l}$ of $1 \times$ 'immunoprecipitation buffer' (25 mM Hepes pH 7.5, $1 \%$ Brij 97, 150 $\mathrm{mM} \mathrm{NaCl}, 5 \mathrm{mM} \mathrm{MgCl}_{2}, 0.2 \%$ SDS and protease inhibitors). After a 2 hour incubation at $4^{\circ} \mathrm{C}, \mathrm{J} 17$ antiserum against BP180 was added to a $1: 100$ dilution and incubated at $4^{\circ} \mathrm{C}$ for a further 2 hours. Subsequently, $50 \mu \mathrm{l}$ of protein $\mathrm{G}$ agarose (Gibco/BRL, Gaithersburg, $\mathrm{MD}$ ) were added to the mix for an additional 1 hour. The protein $\mathrm{G}$ agarose was collected by centrifugation, washed four times in buffer and then solubilized in sample buffer. The resulting protein solution was processed for western immunoblotting as detailed above.

In the case of pp126 cells, extracts were prepared using immunoprecipitation buffer, which was then processed as described above, with one exception, the extracts were passed through a 22 gauge needle prior to addition of antibody.

\section{Immunofluorescence microscopy}

Cells, grown on glass coverslips, were fixed for 1 minute in $3.7 \%$ formaldehyde and then extracted in $0.5 \%$ Triton $\mathrm{X}-100$ at $4{ }^{\circ} \mathrm{C}$ for 7 minutes. Single and double-label immunofluorescence were performed as detailed previously (Riddelle et al., 1992). After mounting, coverslips were viewed on a Zeiss LSM510 confocal microscope, fitted with appropriate filters for visualization of GFP as well as fluorescein and rhodamine-conjugated probes (Carl Zeiss, Thornwood, NY). Controls for immunocytochemistry included omission of primary antibodies or use of irrelevant immunoglobulin (IgG) to determine non-specific binding of secondary antibodies.

Oral tissues were rapidly frozen in liquid nitrogen and cryosections 5-6 $\mu \mathrm{m}$ thick, were prepared on a Tissue-Trek cryostat (Miles Laboratories Inc., Elkhart, IN). Sections were then placed on microscope slides and fixed for 5 minutes in acetone at $-20^{\circ} \mathrm{C}$. Samples were air-dried and processed for double-label immunofluorescence as described above.

\section{Antibody blocking assays}

A polyclonal antibody against the C-terminal extracellular domain of BP180 was added to pp126 cells maintained in low calcium medium at a final concentration of $50 \mu \mathrm{g} / \mathrm{ml}$ for 24 hours. Rabbit IgG serum was used as a control. Calcium from a 100X stock was added to each dish to a final calcium concentration of $2.0 \mathrm{mM}$ in the presence of BP180 antibody or control IgG. After 4 hours, cells were processed for immunofluorescence as described above. Rabbit IgG was visualized using a fluorochrome-conjugated secondary anti-rabbit goat antibody.

\section{RESULTS}

\section{BP180 interacts with actinin family members}

In this study, it was our initial goal to use the yeast two-hybrid technique to identify novel binding partners of the cytoplasmic domain of the BP180 molecule. To do so, a library was constructed using MCF10A cell cDNA cloned into a pGAD vector containing the GAL4-activating domain. We chose MCF-10A cells for this purpose since they express all of the known components of hemidesmosomes and assemble mature hemidesmosomes in vitro (Stahl et al., 1997). A BP180 cDNA encoding amino acid residues 1-517, cloned into the pAS2-1 vector containing the GAL4 DNA binding domain, was used as 'bait'.

The bait and the MCF-10A cDNA library were cotransfected into the Y190 yeast strain and the transfected yeast were plated onto restrictive media. Yeast colonies able to grow on the media were analyzed further for $\beta$-galactosidase activity. Approximately 20 clones that turn blue were characterized at the molecular level. One such clone contained a cDNA insert encoding residues 708-813 of an actinin isoform termed actinin-4 (Honda et al., 1998) (Table 1). We decided to focus on this clone because we were intrigued by the possibility that BP180 might interact with the actin cytoskeleton. To confirm that BP180 and actinin-4 specifically interact in the yeast twohybrid assay, we prepared an actinin- 4 cDNA encoding the same residues (703-813) by RT-PCR using mRNA prepared from MCF-10A cells. This cDNA was cloned into a pACT2 yeast expression vector. Following co-transfection with a pAS2-1 vector containing a BP180 cDNA, the transfected yeast grow on restrictive medium and turn blue in the $\beta$ galactosidase assay (Table 1). Control transfectants including those co-expressing either actinin-4 or BP180 in combination with $\alpha 6$ integrin, p53 or large T-antigen do not grow on $\mathrm{Leu}^{-} / \mathrm{Trp}^{-} / \mathrm{His}^{-}$medium and fail to turn blue under the same conditions (Table 1).

Our yeast two-hybrid assay reveals that a fragment of 110 amino acids in the actinin-4 molecule can interact with the cytoplasmic domain of BP180. This fragment contains two EFhand domains and shows considerable homology with other actinin family members (Fig. 1). We therefore tested the possibility that actinin-1, an actinin isoform expressed by epithelial cells, can interact with BP180 (Otey et al., 1990). An actinin-1 cDNA encoding residues 708-850 was generated by RT-PCR using mRNA isolated from MCF-10A cells and

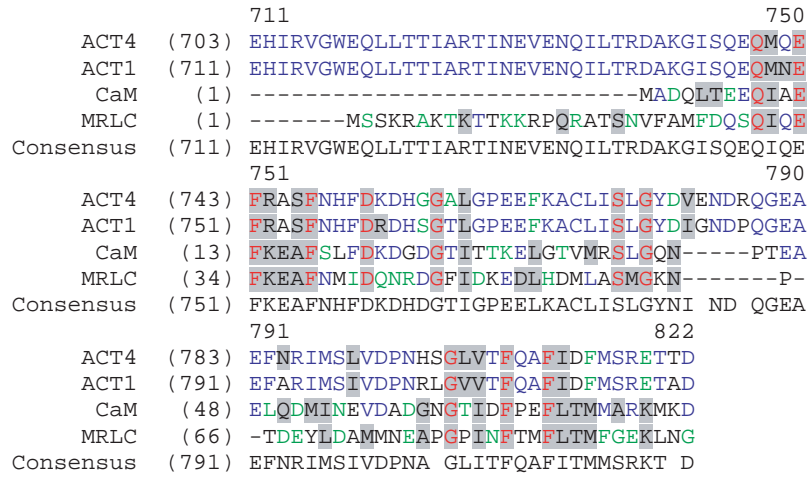

Fig. 1. Amino acid comparison of actinin-4 and other EF-hand proteins. The region in actinin-4 that interacts with BP180 is conserved among actinin family members and contains two EF-hand domains. The schematic includes a comparison of the BP180-binding sequence in actinin-4 and the comparable EF-hand domains in actinin-1, calmodulin and myosin regulatory light chain (MRLC). 


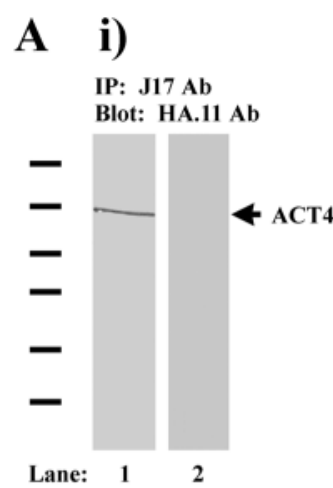

B i)

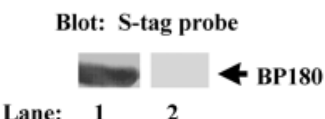

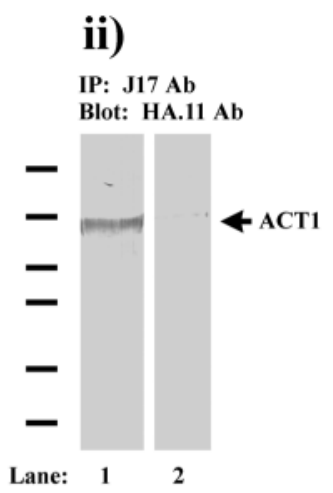

ii)

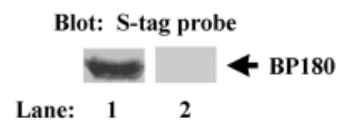

iii)

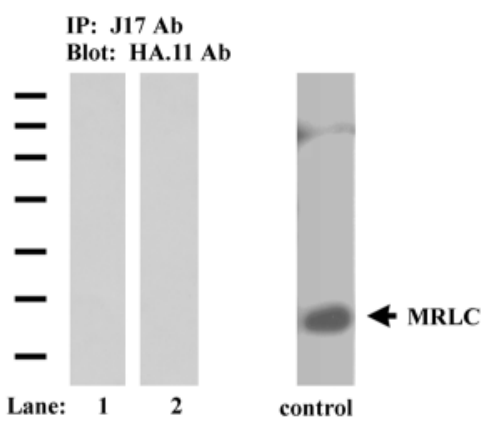

iii)

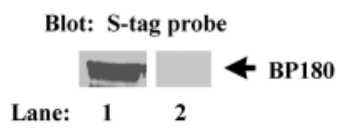

Fig. 2. Immunoprecipitation studies indicate that BP180 can bind actinin- 4 and actinin- 1 but not MRLC. Approximately $1 \mu \mathrm{g}$ each of either HA-tagged actinin-4 (Ai), actinin-1 (Aii) or MRLC (Aiii) were incubated with a purified S-tagged BP180 fusion polypeptide in Hepes-buffered saline with $1 \%$ Brij 97 and $0.2 \%$ SDS at $4{ }^{\circ} \mathrm{C}$ for 2 hours. As a control, tagged BP180 polypeptide was omitted from the immunoprecipitation mixture (Ai-iii, lane 2). A polyclonal antiserum against the BP180 cytoplasmic domain was added to the protein mixture for an additional 2 hours, followed by the addition of protein G-agarose beads (Life Technologies/BRL). The precipitated proteins were subjected to SDS-PAGE and transferred to nitrocellulose membrane. The blot was probed either with HA.11 antibody directed against the HA tag (A) or an alkaline phosphatase-conjugated S-protein that binds S-tag peptide fusion proteins (B). In lane 1 in Ai, the HA.11 antibody recognizes a single polypeptide, indicating that actinin-4 has been precipitated with BP180. Similar results were obtained when BP180 was precipitated from the BP180/actinin-1 mix (lane 1 in Aii). By contrast, MRLC was not precipitated from the MRLC/BP180 mix as indicated by the absence of a band in lane 1 in Aiii. No protein is recognized by the HA.11 antibody in Ai-iii lane 2 , indicating that actinin- 4 , actinin- 1 or MRLC were not precipitated by the BP180 antiserum. The enzyme-linked S-protein in B recognizes the immunoprecipitated BP180 polypeptide in Bi-iii lane 1. Molecular mass markers indicated on the left of the upper panels are as follows: from top to bottom $172,111,79,61,49,36 \mathrm{kDa}$.

cloned into the pACT2 vector. Yeast co-transfected with this actinin-1 construct and BP180/pAS2-1 grow in restrictive media and turn blue in a $\beta$-galactosidase assay (Table 1$)$.

Because the region of actinins that shows interaction with BP180 contains two EF-hand domains, we next evaluated the possibility that the EF-hand domain possesses a consensus- binding site for BP180. To do so, we tested whether proteins that contain different numbers of EF-hand domains show interaction with BP180 in the yeast two-hybrid system (Table 1). We chose calmodulin, which contains four such domains, and myosin regulatory light chain (MRLC) with one EF hand domain for these assays. Both calmodulin and MRLC were cloned into the pACT2 vector and transfected into yeast cells containing the BP180/pAS2-1 construct. The co-transfectants are unable to grow on restrictive media and do not turn blue in the $\beta$ galactosidase assay indicating that there is no interaction between BP180 and the EF-hand domains of either calmodulin or MRLC (Table 1).

To provide additional evidence for interaction between actinin family members and BP180, we prepared recombinant proteins of full-length actinin-4, actinin-1, MRLC and the cytoplasmic fragment (residues 1461) of BP180 in bacteria. All proteins were His-tagged and were purified by column chromatography. In addition to the His tag, the recombinant actinin-4, actinin-1 and MRLC were tagged with a hemagglutinin (HA) epitope that is recognized by monoclonal antibody HA.11. The BP180 fragment was fused to an S-tag peptide that binds ribonuclease S-protein. The actinin-4, actinin-1 or MRLC and BP180 recombinant proteins were mixed in Hepes-buffered saline containing a cocktail of protease inhibitors. Proteins were precipitated by the addition of a 1:100 dilution of antiserum $\mathrm{J} 17$, which was generated against the N-terminus of BP180 (Hopkinson et al., 1992).

Table 1. Identification of positive clones by yeast two-hybrid

\begin{tabular}{|c|c|c|}
\hline Test pairs & $\begin{array}{l}\text { Plating efficiency on } \mathrm{Leu}^{-} / \mathrm{Trp}^{-} / \mathrm{His}^{-} \\
\quad+25 \mathrm{mM} 3 \text {-AT medium }\end{array}$ & $\beta$-gal activity \\
\hline BP180 ${ }^{1-517} \mathrm{pAS} 2-1 / \mathrm{ACT}^{703-813}$ pGAD & +++ & +++ \\
\hline $\mathrm{BP} 180^{1-517} \mathrm{pAS} 2-1 / \mathrm{ACT} 4^{703-813}$ pACT2 & +++ & +++ \\
\hline$\alpha 6$ Integrin full length pAS2-1/ACT4 $4^{703-813}$ pACT2 & - & - \\
\hline p53 pAS2-1/ACT4 ${ }^{703-813}$ pACT2 & - & - \\
\hline BP180 $0^{1-517} \mathrm{pAS} 2-1 /$ large T-antigen $\mathrm{pACT} 2$ & - & - \\
\hline BP180 $0^{1-517} \mathrm{pAS} 2-1 / \mathrm{ACT} 1^{708-850} \mathrm{pACT} 2$ & +++ & +++ \\
\hline p53 pAS2-1/ACT $1{ }^{708-850}$ pACT2 & - & - \\
\hline BP180 $1-517$ pAS2-1/CaM pACT2 & - & - \\
\hline BP1801-517 $\mathrm{pAS} 2-1 / \mathrm{MRLC}$ pACT2 & - & - \\
\hline
\end{tabular}

Yeasts transfected with the indicated yeast constructs were plated on both $\mathrm{Leu}^{-} / \mathrm{Trp}^{-}$and $\mathrm{Leu}^{-} / \mathrm{Trp}^{-} / \mathrm{His}^{-}+25 \mathrm{mM} 3-\mathrm{AT}$ medium. Yeast were allowed to grow for 7 days. Plating efficiency on $\mathrm{Leu}^{-} / \mathrm{Trp}^{-} / \mathrm{His}^{-}+25 \mathrm{mM} 3$-AT medium is expressed relative to that on $\mathrm{Leu}^{-} / \mathrm{Trp}^{-}$medium. +++ and - indicate plating efficiencies of $>50 \%$ and $0 \%$, respectively. Colonies that grew on Leu $/ \mathrm{Trp}^{-} / \mathrm{His}^{-}+25 \mathrm{mM}$ 3-AT medium were transferred to nylon filters. These filters were then placed on X-gal-soaked filters, and the ability of yeast to turn blue was assessed. In instances where there was no growth in Leu ${ }^{-} / \mathrm{Trp}^{-} / \mathrm{His}^{-}+25 \mathrm{mM}^{-}$-AT medium, colonies from Leu-/Trp ${ }^{-}$medium were analyzed. +++ , colonies that turned blue within 6 hours of development; - , colonies that failed to turn blue within this time period. Each pair was tested at least three times for plating efficiency on Leu $^{-} / \mathrm{Trp}^{-} / \mathrm{His}^{-}+25 \mathrm{mM}^{-}$-AT medium and in the $\beta$-galactosidase assay. 


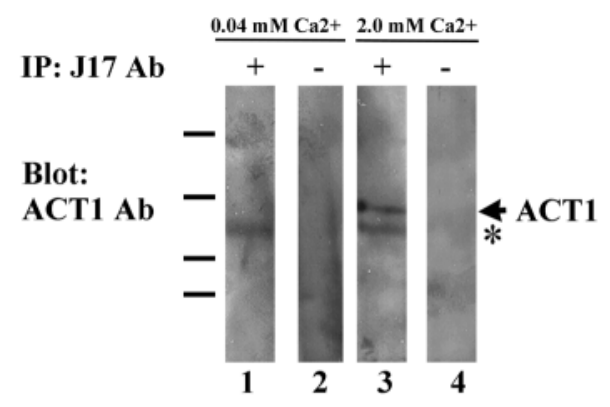

Blot:

J17 Ab

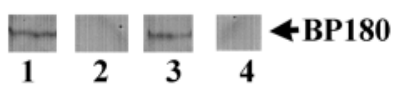

Fig. 3. BP180 can co-precipitate actinin- 1 from pp126 cells. Extracts of pp126 cells were prepared from cells maintained in medium containing low calcium (lanes 1,2 ) or cells which had been switched into medium containing $2.0 \mathrm{mM}$ calcium for 4 hours (lanes 3,4). A polyclonal antiserum against the BP180 cytoplasmic domain (J17) (lanes 1,3) was added to the cell extracts which were incubated for 2 hours. No antiserum was included in the extracts shown in lanes 2 and 4 . Subsequently protein $\mathrm{G}$-agarose beads were added and, after an additional 2 hours, the beads collected.

Precipitated proteins were subjected to SDS-PAGE, transferred to nitrocellulose membranes and processed for immunoblotting using an actinin-1 monoclonal antibody (top). The same immunoblot was then probed with the BP180 antiserum to ensure that BP180 protein had been successfully precipitated (bottom). Note that actinin-1 at $100 \mathrm{kDa}$ is precipitated with BP180 in lane 3 only. An unknown protein recognized by the actinin-1 probe is indicated by an asterisk in lanes 1 and 3 in the top panels. Molecular mass markers indicated on the left of the upper panels are as follows: from top to bottom: 172, 111, 79, $61 \mathrm{kDa}$.

Precipitated proteins were separated by SDS-PAGE, and transferred to nitrocellulose. The filter was probed with HA.11 antibodies, which recognize the HA epitope on the actinin-4, actinin-1 or MRLC polypeptides. Actinin-4 is co-precipitated by the J17 antiserum when mixed with purified BP180 cytoplasmic domain but is not co-precipitated by $\mathrm{J} 17$ antibodies when incubated in the absence of BP180 protein (Fig. 2Ai, compare lanes 1 and 2). Similar results were obtained when actinin-1 was mixed with the BP180 fragment (Fig. 2Aii). By contrast, MRLC is not co-precipitated by the J17 antiserum when mixed with purified BP180 cytoplasmic domain and is not co-precipitated by J17 antibody when incubated in the absence of BP180 protein (Fig. 2Aiii). Sprotein binding was used to confirm successful precipitation of BP180 polypeptide (Fig. 2B).

\section{Actinin-1 co-precipitates with BP180 from pp126 cells}

To provide evidence that BP180 and actinin family members associate in vivo, we undertook immunoprecipitation studies (Fig. 3). Extracts of pp126 oral keratinocyte cells were prepared from cells maintained in medium containing reduced levels of calcium or cells 'switched' to medium containing $2 \mathrm{mM}$ calcium for 4 hours. Antibodies against BP180 specifically precipitate actinin-1 from extracts of cells maintained in medium containing $2 \mathrm{mM}$ calcium but fail to precipitate actinin-1 from pp126 cells from cells grown in low calcium medium (Fig. 3). By contrast, actinin-4 is not precipitated by BP180 antibodies from extracts of pp126 cells regardless of medium conditions (not shown).

\section{Actinin family members co-distribute with BP180 at sites of cell-cell contact}

Actinin-4, actinin-1 and BP180 fail to localize at regions of cell-cell interaction in pp126 oral keratinocytes when the cells are maintained in medium containing reduced levels of calcium (Fig. 4). Actinin-4 shows concentration at the cell edges in ruffled membranes while actinin-1 localizes to focal contacts at the base of the cell and cortical microfilament arrays (Fig. $4 \mathrm{~A}, \mathrm{~B})$. By contrast, BP180 is found in streaks and arcs at the basal surface of the cells (Fig. 4C). In pp126 cells that have been 'switched' to medium containing $2 \mathrm{mM}$ calcium for 4 hours prior to processing for immunofluorescence microscopic observation, all three proteins appear at cell-cell contact sites (Fig. 5). Moreover, in pp126 cells under these conditions BP180 co-localizes with both actinin-4 as well as actinin-1 at such sites (Fig. 5A-C and D-F, respectively). BP230 and plectin, two hemidesmosome proteins, fail to localize at sites of cell-cell contact in high calcium but instead distribute at the basal aspect of cells where they adhere to substratum (not shown).

We have compared the localization of BP180 with both actinin-1 and actinin-4 in a second epithelial cell line. In MCF$10 \mathrm{~A}$ cells, BP180 stains in arcs and streaks at the substratum attached surface of the cells (Fig. 6B,E, long arrow). Along this surface of the cell there is no obvious co-distribution of BP180 with either actinin-1 or actinin-4. However, BP180 also shows concentration along regions of cell-cell contact consistent with a previous report (Kitajima et al., 1994) (Fig. 6B,E, small arrows). We observe such intercellular BP180 distribution with two different antibody probes against the N-terminus of BP180. Actinin-4 and actinin-1 also localize at sites of cellcell interaction and their distribution overlaps with that of BP180 (Fig. 6A,B). This is most clearly seen in the overlays
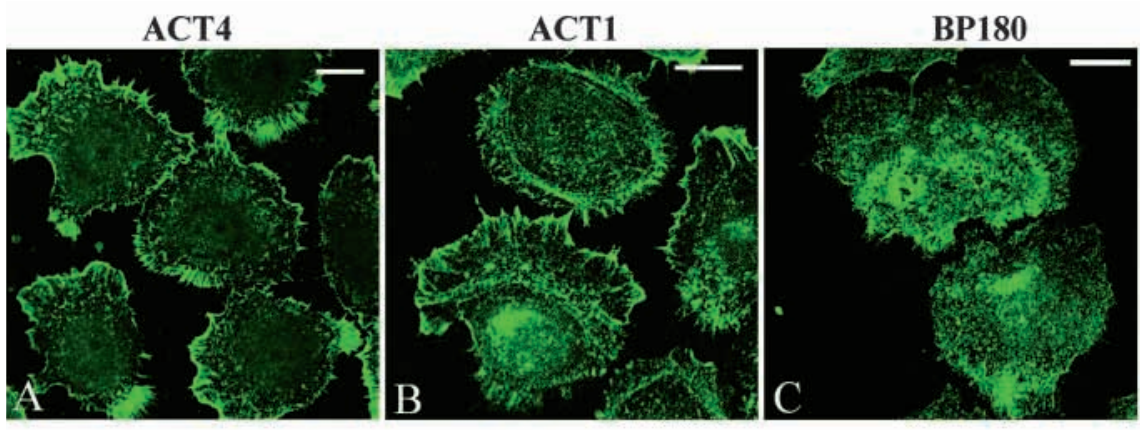

Fig. 4. Sub-cellular distribution of actinin-4, actinin-1 and BP180 in pp126 oral keratinocytes maintained in low calcium medium. Cells were processed for immunofluorescence microscopy and labeled with antibodies against actinin-4 (A), actinin-1 (B) and BP180 (C). Actinin-4 localizes to the cell-edge in membrane ruffles in A while actinin- 1 is present in focal contact-like structures and along the cortical microfilament cytoskeleton in B. By contrast, BP180 localizes along the basal aspect of the cell (C). Bars, $10 \mu \mathrm{m}$. 


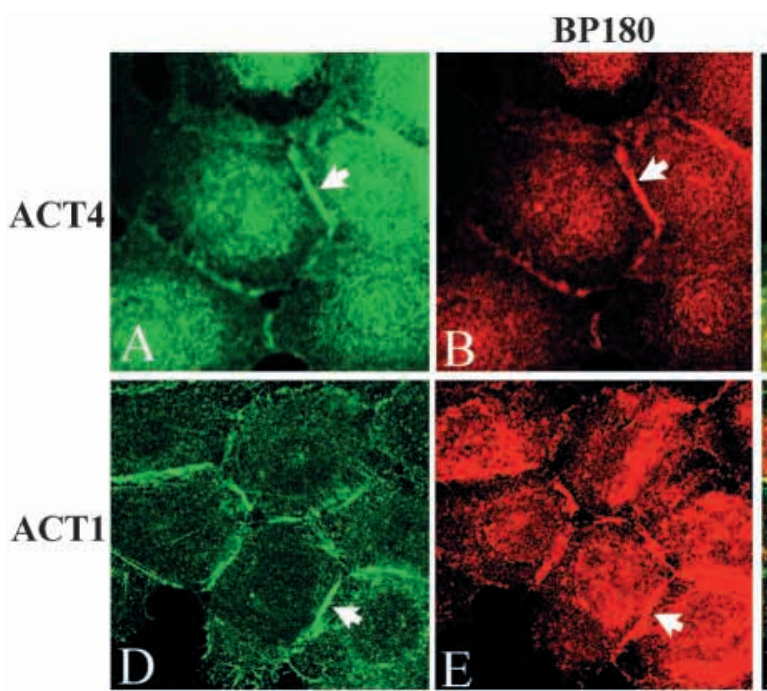

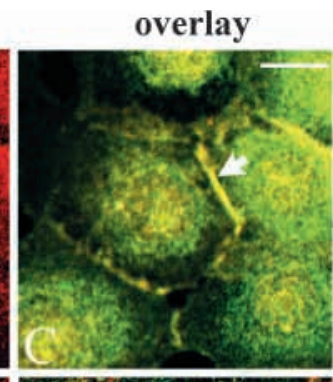

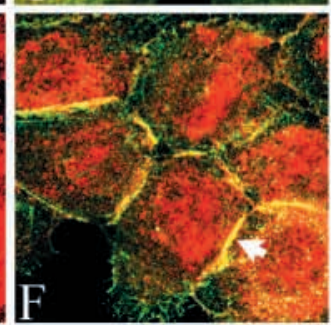

Fig. 5. Increasing the concentration of calcium ions in the medium induces co-localization of BP180, actinin- 4 and actinin- 1 at cell-cell contact sites in pp126 oral keratinocytes. pp126 oral keratinocytes were 'switched' to medium containing $2.0 \mathrm{mM}$ calcium for 4 hours and then were processed for double-label indirect immunofluorescence microscopy using a combination of antibodies against actinin-4 and BP180 (A,B) or actinin-1 and BP180 (D,E). Both actinins and BP180 co-distribute at cell-cell contact sites (arrows in A-E). The overlapping staining patterns appear yellow in the overlays $(\mathrm{C}, \mathrm{F})$. Bar, $10 \mu \mathrm{m}$. of the staining pattern where cell borders appear yellow where BP180 co-distributes either with actinin-4 or actinin-1 (Fig. 6C and $\mathrm{F}$, respectively).

Our immunofluorescence observations provide evidence that actinin- 1 and actinin- 4 are found at the cell surface in two distinct epithelial cell populations (Fig. 4; Fig. 6). By contrast, workers in a previous study suggested that actinin-4 localizes exclusively to the nucleus in certain breast epithelial cells (Honda et al., 1998). Thus to resolve these conflicting results we transfected MCF-10A cells with an expression vector encoding GFP-tagged actinin-4. The product of the transgene localizes to sites of cell-cell interaction on the surface of the transfected cells (Fig. 7A). We see little, if any, protein in the nucleus. To rule out the possibility that the GFP tag influences the localization of actinin-4, we also transfected MCF-10A cells with HA-tagged actinin-4 (Fig. 7C). The expressed fusion protein targets to the cell surface rather than the nucleus. Interestingly, we have observed only nuclear localization of actinin-4 when we induce expression of a truncated HA-tagged actinin-4 cDNA encompassing residues 1-813 in MCF-10A cells (Fig. 7E).

Our finding that under certain circumstances, BP180 is present at sites of cell-cell contact, where it associates with adherens junction proteins, is consistent with a study in which it was shown using yeast two-hybrid technology that BP180 can bind the adherens junction protein p120 catenin (Aho et al., 1999). The latter workers, however, provided no evidence for such an association at the morphological level. Thus to extend their observation we prepared both MCF-10A and pp126 cells for double-label immunofluorescence microscopy using antibodies against the $\mathrm{N}$-terminal domain of BP180 and monoclonal antibodies against p120 catenin (Fig. 8). These antibody preparations generate overlapping staining patterns at cell-cell contact sites in both cell types (Fig. 8). The overlay images in Fig. 8C,F show yellow staining where cells contact each other. In the case of the pp126 cells, the cells were switched from their normal medium to medium containing $2 \mathrm{mM}$ calcium for 4 hours before the cells were fixed and processed for immunofluorescence in order to allow cells to establish cellcell junctions. Moreover, we also processed oral tissues for double-labeling with antibodies that recognize BP180 and p120 catenin. Antibodies against BP180 produced intense staining along the basement membrane of oral epithelia. p120 catenin was absent from these sites (Fig. 9, arrow). However, BP180 was also present at cell-cell borders where it codistributed with p120 catenin (Fig. 9, arrowhead). Similar

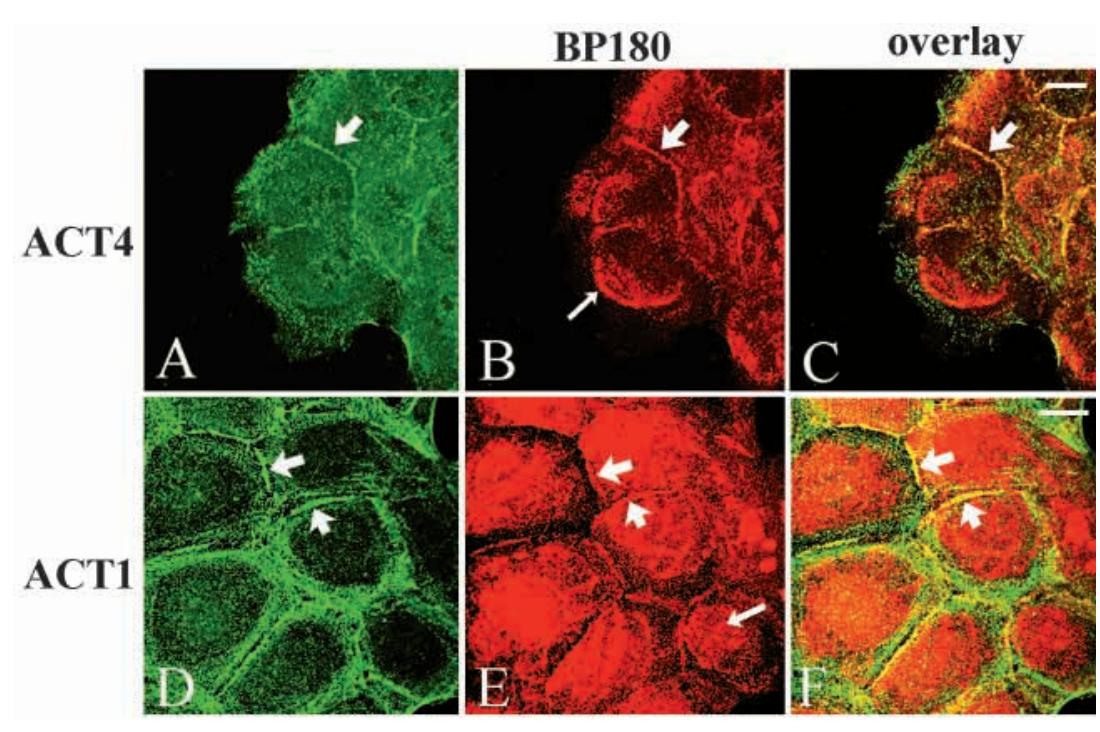

Fig. 6. BP180 co-distributes with actinin- 4 and actinin- 1 at cell-cell contact sites in MCF-10A cells. MCF-10A cells were processed for double-labeling using antibodies against either actinin-4 (A) or actinin-1 (D) in combination with an antibody preparation against BP180 (B,E). BP180 co-localizes with actinin-4 at sites of cellcell contact (arrows in A,B). This appears yellow in the overlay of these images (C). Actinin-1 and BP180 also co-distribute at cell-cell contact sites (arrows in D,E). The areas of co-localization appear yellow in the overlay of the images $(\mathrm{F})$. There is no obvious actinin- 4 or actinin-1 where BP180 is found along sites of cell-substrate interaction (B,E, long arrows). Bar, $10 \mu \mathrm{m}$. 


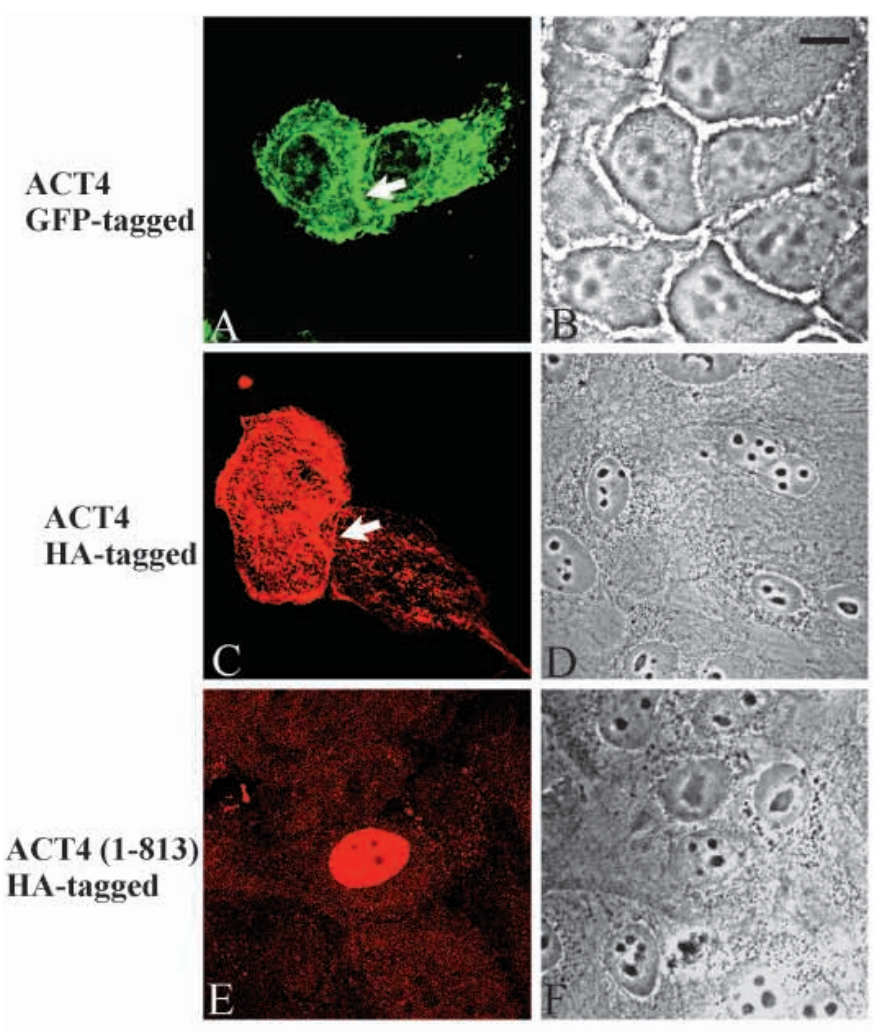

observations were obtained with actinin probes (data not shown).

\section{Does BP180 play a role in cell-cell interactions?}

Our studies demonstrate that BP180 is present in cell-cell contacts and we next wished to determine whether BP180 plays a functional role at such sites. Thus we made use of an antibody that recognizes the extracellular domain of BP180 in an attempt to block BP180's function. pp126 cells maintained in low calcium were pretreated with a C-terminal antibody against BP180. After 24 hours, cells were switched to high calcium for 4 hours and labeled with either p120 catenin or E-cadherin monoclonal antibodies as markers of adherens
Fig. 7. The fate of the product of actinin-4 transgenes in transfected MCF-10A cells. MCF-10A cells were transfected with constructs expressing full-length actinin-4 fused with a GFP-tag (A) or an HA$\operatorname{tag}(\mathrm{C})$. In E, cells were transfected with a truncated actinin-4 (residues 1-813). At 24 hours after transfection, cells were processed for immunofluorescence microscopy. HA.11 antibody was used to visualize HA-tagged actinin-4 in transfected cells in C,E. Full-length actinin-4 is absent from the nucleus but instead distributes to sites of cell-cell contact as well as in the cytoplasm (arrows in A,C). By contrast, truncated actinin-4 localizes exclusively to the nucleus (E). $\mathrm{B}, \mathrm{D}$ and $\mathrm{F}$ show phase contrast images of the cells. Bar, $10 \mu \mathrm{m}$.

junctions. In untreated cells and cells treated with control immunoglobulin, p120 catenin and E-cadherin have a sharp, linear distribution along borders of contacting cells (Fig. $10 \mathrm{~A}, \mathrm{~B}$, arrow). By contrast, p120 catenin fails to show the same pattern in cells that were treated with the C-terminal antibody against BP180 (Fig. 10C, arrow). Rather, in BP180 antibody treated cells, p120 catenin is organized in arrays that are perpendicular to cell-cell contact sites. In BP180 antibody treated cells, E-cadherin is diffusely localized (Fig. 10E).

\section{DISCUSSION}

BP180 is a major structural element of hemidesmosomes where it associates with not only the $\alpha 6 \beta 4$ integrin heterodimer but also the BP230 component of the hemidesmosome plaque (Borradori and Sonnenberg, 1999; Jones et al., 1998; Hopkinson and Jones, 2000). BP180 mediates keratin cytoskeleton association with the cell surface in epithelial cells via such interactions (Borradori and Sonnenberg, 1999; Jones, et al., 1998; Hopkinson and Jones, 2000). Therefore, it was a surprise that during the course of the present studies we identified two microfilament associated proteins, namely actinin-1 and actinin-4, as binding partners of BP180. Nonetheless, our immunochemical studies clearly support the idea that BP180 and actinin family members show association in vivo. Indeed, we have observed overlapping staining patterns of antibodies against BP180 and actinin family members at sites of cell-cell contact in two quite different epithelial cells

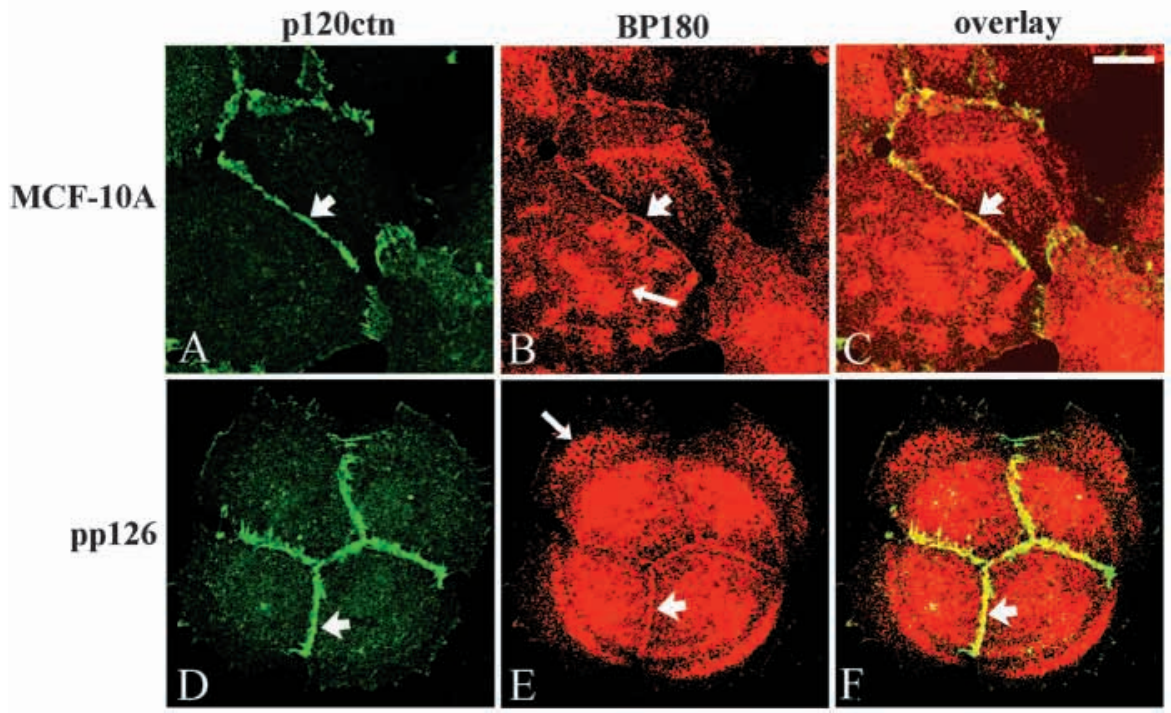

Fig. 8. BP180 co-distributes with p120 catenin in MCF-10A cells and pp126 oral keratinocytes. The localization of p120 catenin and BP180 proteins was assessed in MCF-10A cells and pp126 oral keratinocytes maintained in $2.0 \mathrm{mM}$ calcium for 4 hours. Cells were processed for double-label indirect immunofluorescence microscopy using antibodies against p120 catenin (A,D) and $\mathrm{BP} 180(\mathrm{~B}, \mathrm{E})$. p120 catenin is present at cellcell interaction sites in both cell types (short arrows in A,D) where it co-distributes with $\mathrm{BP} 180$ (short arrows in B,E). This is demonstrated by the yellow color in the overlays in C and F. Although BP180 antibodies also stain along the basal aspect of MCF-10A cells (long arrow in B) and in pp126 cells (long arrow in E), p120 catenin is absent from these sites (A,D). Bar, $10 \mu \mathrm{m}$. 


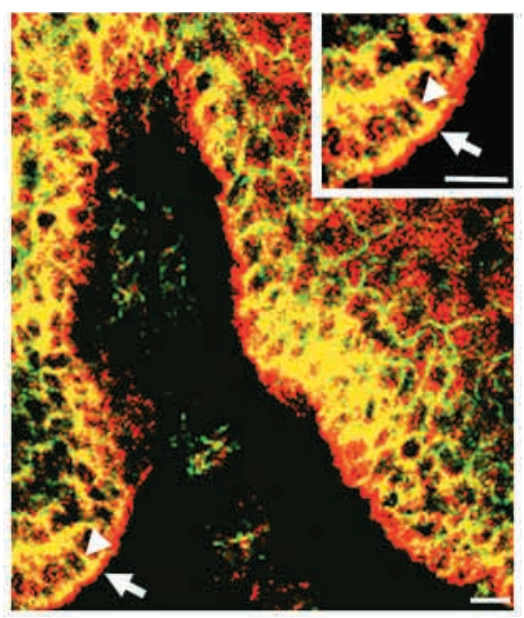

Fig. 9. BP180 co-distributes with p120 catenin, at sites of cell-cell contact in oral tissue. Tissues were processed for double-label indirect immunofluorescence microscopy using antibodies against BP180 (in red) and p120 catenin (in green). The overlay of the images generated is shown. The area indicated by the arrows toward the left-hand corner of the micrograph is shown at higher power in the inset. BP180 distributes along the basement membrane (arrow) and along lateral surfaces of basal oral epithelial cells (arrow head) where it co-distributes with p120 catenin as demonstrated by the yellow color. Bars, $20 \mu \mathrm{m}$.

types, namely the oral keratinocyte line pp126 and the mammary epithelial cell line MCF-10A, as well as in oral epithelial tissue. This is not the first time that the existence of BP180 at cell-cell binding sites has been reported. In particular, Kitajima et al. showed that in keratinocytes the localization of BP180 can be modulated by the concentration of calcium of the medium in which the cells are maintained (Kitajima et al., 1994). We have confirmed and extended this observation. In medium containing low levels of calcium, under which conditions keratinocytes fail to make extensive cell-cell contacts, BP180 localizes at the basal aspect of the cell at cellmatrix contact sites. Both actinin- 1 and actinin- 4 fail to codistribute with BP180 at these sites. These morphological observations are supported by immunoprecipitation studies since BP180 antibodies fail to precipitate actinins from extracts of such cells. However, upon 'switching' the cells to medium containing calcium levels of $2 \mathrm{mM}$, cell-cell junctions form with BP180 distributing to these sites along with actinin-1 and actinin-4 and to regions of cell-matrix interaction that lack any apparent actinin. Moreover, BP180 antibodies precipitate actinin-1 from extracts of the switched cells. That actinin- 4 is not precipitated under these conditions suggests that actinin-1 may be the preferred BP180 binding partner in vivo.

One surprise of our study was that actinin-4, like actinin-1, is present at cell-cell contact sites both in MCF-10A cells and the 'switched' oral keratinocytes. This finding is inconsistent with a previous study by Honda et al. in which it was reported that actinin-4 is a nuclear protein (Honda et al., 1998). To address this apparent discrepancy we expressed full-length actinin-4 in our epithelial cell populations. This confirmed our antibody labeling experiments in that the protein product of the actinin-4 transgene primarily targets to the cell surface and is absent from the nucleus.

Interestingly, as part of our analyses of actinin-4 distribution
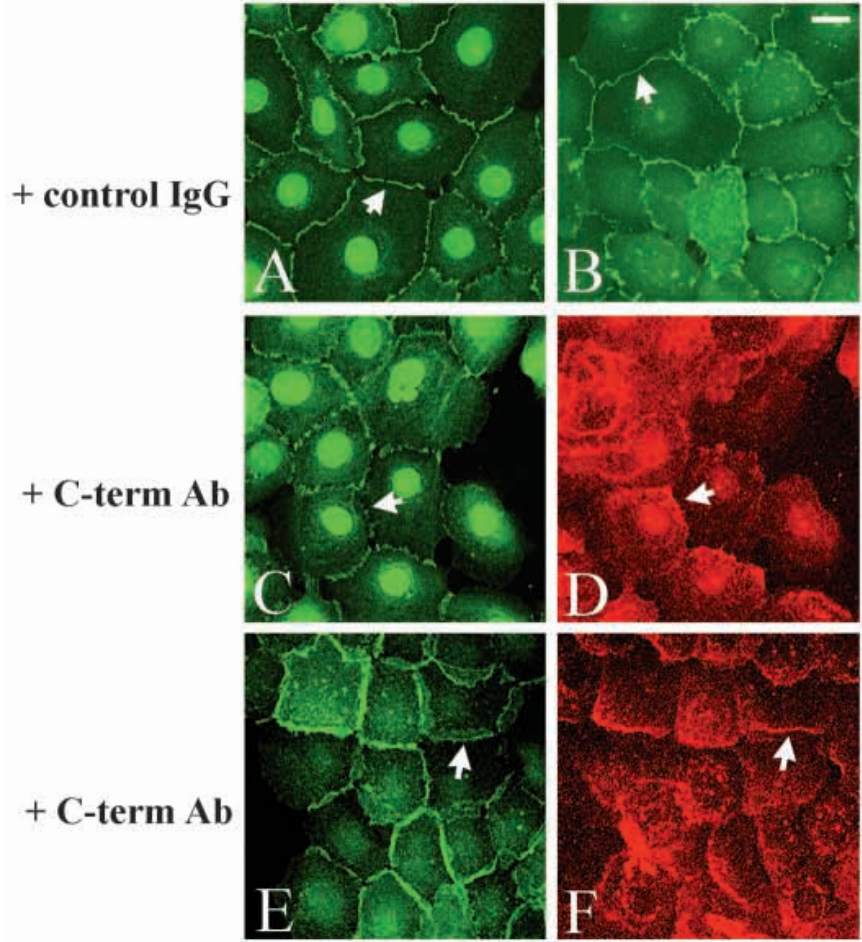

Fig. 10. BP180 modulates establishment of cell-cell junctions. pp126 cells maintained in low calcium medium were treated with either control IgG or an antibody against the C-terminus of BP180 for 24 hours and then switched to medium containing $2.0 \mathrm{mM}$ calcium. After 4 hours, cells were processed for confocal immunofluorescence microscopy using an antibody against p120 catenin $(\mathrm{A}, \mathrm{C})$ or Ecadherin $(\mathrm{B}, \mathrm{E})$ together with a secondary antibody to identify bound BP180 antibodies (D,F). In cells treated with control IgG, p120 catenin and E-cadherin staining is linear and is concentrated along sites of cell-cell contact (A,B). In cells treated with the BP180 antibody, p120 catenin appears in a punctate pattern in rows perpendicular to cell-cell contact sites $(\mathrm{C})$, whereas E-cadherin shows a diffuse staining over the cell surface (E). D and $\mathrm{F}$ show that BP180 antibodies bind at cell-cell contact sites. Bar, $10 \mu \mathrm{m}$.

in various cells, we discovered that a truncated version of actinin-4 lacking the last 71 amino acids at the C-terminus of the molecule localizes exclusively to the nucleus. In light of these observations, we suggest that certain cancer cells, including those in which actinin-4 was first described, may process actinin-4, causing it to move to the nucleus (Honda et al., 1998). This would explain the localization data presented by the later study, although, to date, we have been unable to confirm that cancer cells express such a truncated actinin-4 molecule (data not shown).

One question that our study clearly raises relates to why BP180 is found at sites of cell-cell interaction. One possibility is that BP180 molecules at cell-cell borders may be in transit to the basal surface of cells where they become incorporated into hemidesmosomes. In this scenario, the association of BP180 with microfilament-associated protein at lateral cell surfaces may reflect the involvement of cellular motility machinery in BP180 movement between cell membrane domains following its synthesis and processing. At lateral cell surfaces, actinins may provide part of an anchorage site or act as a 'sink' for newly synthesized BP180 protein. In other 
words, actinins may be playing a role as general scaffolding proteins for molecules such as BP180, as previously suggested (Parast et al., 2000).

We also speculate that p120 catenin may play an important role in the mechanism via which BP180 becomes directed to lateral cell surfaces. Previous workers have provided evidence from a yeast two-hybrid screen that BP180 and p120 catenin interact (Aho et al., 1999). Here we show that p120 catenin and BP180 co-distribute at cell-cell contact sites. Since p120 catenin binds to BP180 at a domain that is necessary for the targeting of BP180 to hemidesmosomes, we propose that by binding to BP180, p120 catenin inhibits BP180 incorporation into hemidesmosomes and, together with the actinins, tethers BP180 at cell borders. However, if this is the case, this is not a generalized phenomenon for hemidesmosomal proteins since BP230 or plectin, two cytoplasmic proteins of the hemidesmosomal plaque, are not found at sites of cell-cell contact in pp126 cells that are maintained in medium containing $2.0 \mathrm{mM}$ calcium under which conditions cells assemble extensive intracellular junctions (not shown). Instead, both plectin and BP230 remain concentrated at sites of cellsubstratum interaction in such cells.

Of course, in addition to transiting through lateral cell surfaces, BP180 may actually contribute to the structure and/or stability of cell-cell interactions. There is certainly precedent for this from the studies of other transmembrane, collagenrelated proteins. For example, collagen type XIII, which, like $\mathrm{BP} 180$, is oriented with a type 2 topography in the plasma membrane, is believed to play a role in stabilizing cell-cell junctions (Hagg et al., 1998; Peltonen et al., 1999; Snellman et al., 2000). Moreover, there is evidence that three other type 2 membrane proteins that possess collagen-like extracellular domains, namely, the type 1 macrophage scavenger protein receptor (MSPR), MARCO (a macrophage receptor with collagenous structure) and ectodysplasin are involved in cellcell binding (Elomaa et al., 1995; Ezer et al., 1999; Kodama et al., 1990). In particular, MSPR and MARCO mediate cellbacterial interactions whereas ectodysplasin appears to distribute along cell-cell contact sites, where it interacts with the microfilament cytoskeleton (Elomaa et al., 1995; Ezer et al., 1999; Kodama et al., 1990). Indeed, in the studies we present here we provide evidence that BP180 plays an active role in establishing cell-cell interactions. In particular, an antibody against the C-terminal domain of BP180 appears to inhibit incorporation of adherens junction proteins into assembling junctional complexes.

In summary, we have identified new potential binding partners of a major structural protein of the hemidesmosome. That these partners are members of a family of actin binding proteins that fail to localize to hemidesmosomes provides an indication that BP180 is somewhat promiscuous with regard to its cytoskeleton connections. Remarkably, this mirrors the apparent promiscuity of the $\alpha 6 \beta 4$ integrin heterodimer which, in the hemidesmosome, is involved in tethering the keratin cytoskeleton to the cell surface whereas, in certain cancer cells, it associates with the microfilament cytoskeleton network (Rabinovitz and Mercurio, 1997). Indeed, BP180 is one of an increasing number of proteins whose cytoskeleton allegiance may be modulated in quite a precise fashion and that may play a role in crosstalk of cell-matrix and cell-cell junctions.
This work was supported by grants to J.C.R.J. and A.M.G. (DK60589 and DE12328) from the NIH. We thank Setsuo Hirohashi for generous gifts of antibody, Susan Hopkinson for the yeast library and help in mastering molecular techniques and Rex Chisholm for the MRLC clone. We are grateful for the technical assistance of Xiang He.

\section{REFERENCES}

Aho, S. and Uitto, J. (1998). Direct interaction between the intracellular domains of bullous pemphigoid antigen 2 (BP180) and $\beta 4$ integrin, hemidesmosomal components of basal keratinocytes. Biochem. Biophys. Res. Comm. 243, 694-699.

Aho, S., Rothenberger, K. and Uitto, J. (1999). Human p120ctn catenin: Tissue specific expression of isoforms and molecular interactions with BP180/type XVII collagen. J. Cell. Biochem. 73, 390-399.

Balding, S. D., Diaz, L. A. and Giudice, G. J. (1997). A recombinant form of the human BP180 ectodomain forms a collagen-like homotrimeric complex. Biochem. 36, 8821-8830.

Bedane, C., McMillan, J. R., Balding, S. D., Bernard, P., Prost, C., Bonnetblanc, J. M., Diaz, L. A., Eady, R. A. and Giudice, G. J. (1997). Bullous pemphigoid and cicatricial pemphigoid autoantibodies react with ultrastructurally separable epitopes on the BP180 ectodomain: evidence that BP180 spans the lamina lucida. J. Invest. Dermatol. 108, 901-907.

Borradori, L. and Sonnenberg, A. (1999). Structure and function of hemidesmosomes: more than simple adhesion complexes. J. Invest. Dermatol. 112, 411-418.

Borradori, L., Koch, P. J., Niessen, C. M., Erkeland, S., van Leusden, M. R. and Sonnenberg, A. (1997). The localization of bullous pemphigoid antigen 180 (BP180) in hemidesmosomes is mediated by its cytoplasmic domain and seems to be regulated by the $\beta 4$ integrin subunit. J. Cell Biol. 136, 1333-1347.

Burridge, K. and Chrzanowska-Wodnicka, M. (1996). Focal adhesions, contractility, and signaling. Annu. Rev. Cell Dev. Biol. 12, 463-518.

Elomaa, O., Kangas, M., Sahlberg, C., Tuukkanen, J., Sormunen, R., Liakka, A., Thesieff, I., Kraal, G. and Tryggvason, K. (1995). Cloning of a novel bacteria-binding receptor structurally related to scavenger receptors and expressed in a subset of macrophages. Cell. 80, 603-609.

Ezer, S., Bayes, M., Elomaa, O., Schlessinger, D. and Kere, J. (1999). Ectodysplasin is a collagenous trimeric type II membrane protein with a tumor necrosis factor-like domain and co-localizes with cytoskeletal structures at lateral and apical surfaces of cells. Hum. Mol. Genet. 8, 20792086.

Giudice, G. J., Emery, D. J. and Diaz, L. A. (1992). Cloning and primary structural analysis of the bullous pemphigoid autoantigen, BP-180. J. Invest. Derm. 99, 243-250.

Giudice, G. J., Emery, D. J., Zelickson, B. D., Anhalt, G. J., Liu, Z. and Diaz, L. A. (1993). Bullous pemphigoid and herpes gestationis autoantibodies recognize a common non-collagenous site on the BP180 ectodomain. J. Immunol. 151, 5742-5750.

Goldfinger, L. E., Stack, M. S. and Jones, J. C. R. (1998). Processing of laminin-5 and its functional consequences: role of plasmin and tissue-type plasminogen activator. J. Cell Biol. 141, 255-265.

Hagg, P., Rehn, M., Huhtala, P., Vaisanen, T., Tamminen, M. and Pihlajaniemi, T. (1998). Type XIII collagen is identified as a plasma membrane protein. J. Biol. Chem. 273, 15590-15597.

Harlow, E. and Lane, D. (1988). In Antibodies: A Laboratory Manual. Cold Spring Harbor Laboratory, pp. 92-121. New York: Cold Spring Harbor Laboratory Press.

Hirako, Y., Usukura, J., Nishizawa, Y. and Owaribe, K. (1996). Demonstration of the molecular shape of BP180, a $180-\mathrm{kDa}$ bullous pemphigoid antigen and its potential for trimer formation. J. Biol. Chem. 271, 13739-13745.

Honda, K., Yamada, T., Endo, R., Ino, Y., Gotoh, M., Tsuda, H., Yamada, Y., Chiba, H. and Hirohashi, S. (1998). Actinin-4, a novel actin bundling protein associated with cell motility and cancer invasion. J. Cell Biol. 140, 1383-1393.

Hopkinson, S. B. and Jones, J. C. R. (2000). The $\mathrm{N}$ terminus of the transmembrane protein BP180 interacts with the N-terminal domain of BP230, thereby mediating keratin cytoskeleton anchorage to the cell surface at the site of the hemidesmosome. Mol. Biol. Cell. 11, 277-286.

Hopkinson, S. B., Riddelle, K. S. and Jones, J. C. R. (1992). The 
cytoplasmic domain of the $180 \mathrm{kD}$ bullous pemphigoid antigen, a hemidesmosomal component: molecular and cell biologic characterization. J. Invest. Dermatol. 99, 264-270.

Hopkinson, S. B., Findlay, K. and Jones, J. C. R. (1998). Interaction of BP180 (type XVII collagen) and $\alpha 6$ integrin subunit is necessary for stabilization of hemidesmosome structure. J. Invest. Derm. 111, 1015-1022.

Jones, J. C. R., Hopkinson, S. B. and Goldfinger, L. E. (1998). Structure and assembly of hemidesmosomes. BioEssays. 20, 488-494.

Jonkman, M. F., Jong, M. C. J. M., Heeres, K., Pas, H. H. and van der Meer, J. B. (1995). 180-kD bullous pemphigoid antigen (BP180) is deficient in generalized atrophic benign epidermolysis bullosa. J. Clin. Invest. 95, $1345-1352$.

Kitajima, Y., Hirako, Y., Owaribe, K., Mori, S. and Yaoita, H. (1994). Antibody-binding to the $180-\mathrm{kD}$ bullous pemphigoid antigens at the lateral cell surface causes their internalization and inhibits their assembly at the basal cell surface in cultured keratinocytes. J. Dermatol. 21, 838-846.

Klatte, D. H., Kurpakus, M. A., Grelling, K. A. and Jones, J. C. R. (1989). Immunochemical characterization of three components of the hemidesmosome and their expression in cultured epithelial cells. J. Cell Biol. 109, 3377-3390.

Kodama, T., Freeman, M., Rohrer, L., Zarecky, J., Matsudaira, P. and Krieger, M. (1990). Type I macrophage scavenger receptor contains ahelical and collagen-like coiled coils. Nature 343, 531-535.

Laemmli, U. K. (1970). Cleavage of structural proteins during assembly of the head of bacteriophage T4. Nature 277, 680-685.

Li, K., Tamai, K., Tan, E. M. L. and Uitto, J. (1993). Cloning of type XVII collagen. Complementary and genomic DNA sequences of mouse 180kilodalton bullous pemphigoid antigen (BPAG2) predict an interrupted collagenous domain, a transmembrane segment, and unusual features in the $5^{\prime}$-end of the gene and the 3 -untranslated region of the mRNA. J. Biol. Chem. 268, 8825-8834

Liu, Z., Diaz, L. A., Troy, J. L., Taylor, A. F., Emery, D. J., Fairley, J. A. and Guidice, G. J. (1993). A passive transfer model of the organ-specific autoimmune disease, bullous pemphigoid using antibodies generated against the hemidesmosomal antigen, BP180. J. Clin. Invest. 92, 2480-2488.

Otey, C. A., Pavalko, F. M. and Burridge, K. (1990). An interaction between a-actinin and the b1 integrin subunit in vitro. J. Cell Biol. 111, 721-729.

Parast, T., Mana, M. and Otey, C. (2000). Characterization of palladin, a novel protein localized to stress fibers and cell adhesions. J. Cell Biol. 150, 643-655.

Peltonen, S., Hentula, M., Hagg, P., Yla-Outinen, H., Tuukkanen, J., Lakkakorpi, J., Rehn, M., Pihlajeniemi, T. and Peltonen, J. (1999). A novel component of epidermal cell-matrix and cell-cell contacts: Transmembrane protein type XIII collagen. J. Invest. Dermatol. 113, 635642.

Rabinovitz, I. and Mercurio, A. M. (1997). The integrin $\alpha 6 \beta 4$ functions in carcinoma cell migration on laminin-1 by mediating the formation and stabilization of actin-containing motility structures. J. Cell Biol. 139, 18731884.

Rezniczek, G. A., de Pereda, J. M., Reiper, S. and Wiche, G. (1998) Linking integrin alpha6beta4-based cell adhesion to the intermediate filament cytoskeleton: direct interaction between the beta4 subunit and plectin at multiple molecular sites. J. Cell Biol. 141, 209-226.

Riddelle, K. S., Hopkinson, S. B. and Jones, J. C. R. (1992) Hemidesmosomes in the epithelial cell line 804G: their fate during wound closure, mitosis and drug induced reorganization of the cytoskeleton. J. Cell Sci. 103, 475-490.

Sambrook, J., Fritsch, E. F. and Maniatis, T. (1989). Molecular cloning: a laboratory manual. New York: Cold Spring Harbor Laboratory Press.

Snellman, A., Hongmin, T., Vaisanen, T., Pekka-Kvist, A., Huhtala, P. and Pihlajaniei, T. (2000). A short sequence in the $\mathrm{N}$-terminal region is required for the trimerization of type XIII collagen and is conserved in other collagenous transmembrane proteins. EMBO J. 19, 5051-5059.

Stahl, S., Weitzman, S. and Jones, J. C. R. (1997). The role of laminin-5 and its receptors in mammary epithelial cell branching morphogenesis. $J$. Cell Sci. 110, 55-63. 